\title{
Expansion of Myeloid Derived Suppressor Cells Contributes to Platelet Activation by L-Arginine Deprivation during SARS-CoV-2 Infection
}

\author{
Alessandra Sacchi ${ }^{1}{ }^{*}$, Germana Grassi ${ }^{1}$, Stefania Notari ${ }^{1}$, Simona Gili ${ }^{1}$, Veronica Bordoni ${ }^{1}$, Eleonora Tartaglia ${ }^{1}$, \\ Rita Casetti ${ }^{1}$, Eleonora Cimini ${ }^{1}$, Davide Mariotti ${ }^{1}$, Gabriele Garotto ${ }^{2}$, Alessia Beccacece ${ }^{2}$, Luisa Marchioni ${ }^{2}$, \\ Michele Bibas $^{2}$ (D), Emanuele Nicastri ${ }^{2}$, Giuseppe Ippolito ${ }^{3} \mathbb{D}$ and Chiara Agrati ${ }^{1}$
}

Citation: Sacchi, A.; Grassi, G.; Notari, S.; Gili, S.; Bordoni, V.; Tartaglia, E.; Casetti, R.; Cimini, E.; Mariotti, D.; Garotto, G.; et al. Expansion of Myeloid Derived Suppressor Cells Contributes to Platelet Activation by L-Arginine Deprivation during SARS-CoV-2 Infection. Cells 2021, 10, 2111. https://doi.org/10.3390/cells 10082111

Academic Editor: Mats W. Johansson

Received: 14 July 2021

Accepted: 12 August 2021

Published: 17 August 2021

Publisher's Note: MDPI stays neutral with regard to jurisdictional claims in published maps and institutional affiliations.

Copyright: (c) 2021 by the authors. Licensee MDPI, Basel, Switzerland. This article is an open access article distributed under the terms and conditions of the Creative Commons Attribution (CC BY) license (https:/ / creativecommons.org/licenses/by/ $4.0 /)$.
1 Department of Epidemiology, Pre-Clinical Research and Advanced Diagnostic, National Institute for Infectious Diseases "Lazzaro Spallanzani"-IRCCS, Via Portuense, 292-00149 Rome, Italy; germana.grassi@inmi.it (G.G.); stefania.notari@inmi.it (S.N.); gili.1581732@studenti.uniroma1.it (S.G.); veronica.bordoni@inmi.it (V.B.); eleonora.tartaglia@inmi.it (E.T.); rita.casetti@inmi.it (R.C.); eleonora.cimini@inmi.it (E.C.); davide.mariotti@inmi.it (D.M.); chiara.agrati@inmi.it (C.A.)

2 Clinical Department, National Institute for Infectious Diseases "Lazzaro Spallanzani"-IRCCS, Via Portuense, 292-00149 Rome, Italy; gabriele.garotto@inmi.it (G.G.); alessia.beccacece@inmi.it (A.B.); luisa.marchioni@inmi.it (L.M.); michele.bibas@inmi.it (M.B.); emanule.nicastri@inmi.it (E.N.)

3 National Institute for Infectious Diseases "Lazzaro Spallanzani"-IRCCS, Via Portuense, 292-00149 Rome, Italy; giuseppe.ippolito@inmi.it

* Correspondence: alessandra.sacchi@inmi.it; Tel.: +39-0655170960

\begin{abstract}
Massive platelet activation and thrombotic events characterize severe COVID-19, highlighting their critical role in SARS-CoV-2-induced immunopathology. Since there is a well-described expansion of myeloid-derived suppressor cells (MDSC) in severe COVID-19, we evaluated their possible role in platelet activation during SARS-CoV-2 infection. During COVID-19, a lower plasmatic L-arginine level was observed compared to healthy donors, which correlated with MDSC frequency. Additionally, activated GPIIb/IIIa complex (PAC-1) expression was higher on platelets from severe COVID-19 patients compared to healthy controls and inversely correlated with L-arginine plasmatic concentration. Notably, MDSC were able to induce PAC-1 expression in vitro by reducing L-arginine concentration, indicating a direct role of PMN-MDSC in platelet activation. Accordingly, we found a positive correlation between ex vivo platelet PAC-1 expression and PMN-MDSC frequency. Overall, our data demonstrate the involvement of PMN-MDSC in triggering platelet activation during COVID-19, highlighting a novel role of MDSC in driving COVID-19 pathogenesis.
\end{abstract}

Keywords: COVID-19; MDSC; platelet; L-Arginine

\section{Introduction}

The ongoing COVID-19 pandemic due to the coronavirus SARS-CoV-2 remains a global health emergency. The clinical features of COVID-19 range from asymptomatic to severe pneumonia and fulminant disease [1], but the mechanisms responsible for this wide clinical presentation are not completely clear. In severe COVID-19, coagulation abnormalities appear, inducing a hypercoagulable state and an increased rate of thrombotic and thromboembolic events [2].

The high inflammatory response may contribute to the thrombotic complications by impairing procoagulant-anticoagulant balance, thus facilitating the development of microthrombosis and disseminated intravascular coagulation [3]. Further, the expression of SARS-CoV-2 receptor (angiotensin converting enzyme 2, ACE-2) on platelet membranes suggests a possible direct role of SARS-CoV-2 in platelet activation [4].

It has been shown that arginase I (Arg I) and nitric oxide synthase (iNOS) detract the microenvironment from arginine, inducing platelet activation, and impairing nitric oxide 
synthesis [5]. The myeloid derived suppressor cells (MDSCs) population is one of the main producers of ArgI and iNOS [6]; they strongly expand early after SARS-CoV-2 infection and can predict the fatal outcome of the disease $[7,8]$. MDSCs, defined in humans as HLADR low/- CD11b+ CD14- CD33+ CD15+ (polymorphonuclear, PMN-MDSCs) or HLA-DR low /- CD11b+CD14+CD33+ (monocytic, M-MDSCs) are known to have the remarkable ability to reduce inflammation by suppressing innate and adaptive immune function through several mechanisms, including iNOS, Arg-1, nicotinamide adenine dinucleotide phosphate oxidase (NOX2), and transforming growth factor beta (TGF- $\beta$ ) [9].

In this study, we assessed the capability of PMN-MDSC to activate platelets during SARS-CoV-2 infection. Our results showed a novel role of PMN-MDSC from COVID-19 patients, being able to increase platelet activation by reducing L-arginine concentration, thus contributing to the platelet hyperactivity observed in severe COVID-19.

\section{Materials and Methods}

\subsection{Study Population}

SARS-CoV-2 infected patients $(n=62)$ were treated at the National Institute for Infectious Diseases "Lazzaro Spallanzani" (Rome, Italy). We enrolled SARS-CoV-2 positive patients without other infections such as HIV, HCV, HBV, MTB, and others. Pregnant women were also excluded. All patients were symptomatic, ranging from moderate (PO2/FIO2 $>200, n=31$, no ICU) to severe $(n=31$, requiring intensive care unit admission, ICU). Median age was 65 years (range 22-95), and 60\% were males. ICU and no ICU patients $(63.3 \%$ and $70 \%$, respectively) presented one or more co-morbidities. These included hypertension (ICU $=53.3 \%$, no ICU $=36.7 \%$ ), cardiovascular diseases $(\mathrm{ICU}=26.7 \%$, no ICU $=10 \%)$, obesity $(\mathrm{ICU}=26.7 \%$, no ICU $=23.3 \%)$, diabetes $(\mathrm{ICU}=16.7 \%$, no ICU $=10 \%)$, and cancer (ICU $=10 \%$, no ICU $=16.7)$. Healthy individuals $(\mathrm{HD}, n=9)$ were included as controls.

The study was approved by the institutional review board (approval number: 9/2020) and signed written informed consent was obtained from patients.

\subsection{Plasma Samples Preparation}

Heparin anti-coagulated whole blood samples were centrifuged at $100 \times g$ for $15 \mathrm{~min}$ and platelet rich plasma (PRP) was collected for further use.

\subsection{PBMC and PMN-MDSC Isolation}

Peripheral blood mononuclear cells (PBMC) were isolated from heparin-treated whole blood by density gradient centrifugation (Lympholyte- $\mathrm{H}$, Cederlane, Burlington, ON, USA) PBMC were suspended in RPMI 1640 (Corning Incorporated, NewYork, NJ, USA) and supplemented with $10 \%$ heat-inactivated fetal bovine serum (FBS) (EuroClone, Milan, Italy), penicillin/streptomycin solutions, and $2 \mathrm{mmol} / \mathrm{L}$ L-glutamine (Corning Incorporated, New York, NJ, USA).

PMN-MDSCs were isolated by using CD15 microbeads (MiltenyiBiotec, Bergisch Gladbach, Germany) according to the manufacturer's procedure. Purity was $>90 \%$ as verified by flow-cytometry (data not shown).

\subsection{Platelets-PMN-MDSC Culture}

Purified PMN-MDSCs $\left(2 \times 10^{5}\right)$ were seeded in 96-well plate (Corning-Incorporated, New York, NJ, USA) in the above described medium without FBS. Twenty microliters of PRP from HD were added and cultured at $37^{\circ} \mathrm{C}$. After $4 \mathrm{~h}$, platelet activation was evaluated by flowcytometry, and supernatants were collected for L-Arginine quantification.

\subsection{Flow Cytometry}

Platelet activation was analyzed by using anti-human REAfinity CD41 APC and anti-human REAfinity activated GPIIb/IIIla complex (PAC-1 PE mAb, Bergisch Gladbach, 
Miltenyi Biotec) on ice in the dark. After $15 \mathrm{~min}, 1 \%$ paraformaldehyde was added and samples were acquired by Cytoflex Flow Cytometer (Beckman-Coulter, Brea, CA, USA).

MDSC frequency was evaluated by staining PBMC with customized Duraclon tubes, (FITC-CD11b, ECD-HLA-DR, PC5.5-CD14, PC7-CD33, KrO-CD45, APC-CD80, DRAQ7, APC-alexa750-CD56, APC-alexa750-CD19, APC-alexa750-CD3, Pacific-Blue-CD15, and Beckman-Coulter) following manufacturer's procedures. Data were acquired by CytoFlex flow-cytometer and analyzed by CytExpert (Beckman-Coulter, Brea, CA, USA).

\subsection{L-arginine Quantification}

Plasma samples and co-culture supernatants were centrifuged at $2000 \mathrm{rpm}$ for $10 \mathrm{~min}$ to eliminate platelets and debris. L-arginine level was evaluated by UPLC-MS/MS by using Kairos Amino Acid Kit (Waters, Milford, MA, USA) according to the manufacturer's instruction. Chromatographic separation was performed using an ACQUITY-UPLC system, followed by detection on a Xevo-TQD (Waters, Milford, MA, USA).

\subsection{Statistical Analysis}

GraphPad Prism version 8.00 (GraphPad Software) was used to perform statistical analyses. The non-parametric Kruskal-Wallis with Dunn's post hoc test or the Wilcoxon matched-pairs signed rank test were used. Correlations were evaluated with the nonparametric Spearman test. The $p<0.05$ was considered significant.

\section{Results}

\subsection{Plasmatic L-Arginine in COVID-19 Patients was Correlated to PMN-MDSC Frequency}

We evaluated the plasmatic concentration of L-arginine in patients with moderate (no ICU) and severe (ICU) COVID-19 and HD. A lower plasmatic L-arginine level was observed in both patient groups compared to HD (Figure 1a). Moreover, L-arginine was lower in ICU compared to no ICU patients, suggesting possible association with disease severity. PMN-MDSC percentage was evaluated by flow-cytometry (Figure 1b). A strong negative correlation was found between L-arginine level and PMN-MDSC frequency (Figure 1c), indicating that PMN-MDSC may be involved in the plasmatic L-arginine deprivation during COVID-19. To assess the role of arginine level in driving platelet activation, the expression of PAC-1 on CD41+ platelets was analyzed in a subgroup of SARS-CoV-2 infected patients and HD by flow-cytometry. The representative plots in Fig 1D show PAC-1 expression on platelets from ICU, non ICU patients, and HD. Cumulative analysis shows that the expression of PAC-1 on platelets was higher in ICU patients compared to HD (Figure 1e). In no ICU patients, an intermediate PAC-1 level was observed (Figure 1e), suggesting a higher platelet activation state in more severe patients. The expression of PAC-1 on platelets directly correlated with plasmatic L-arginine (Figure 1f), indicating that arginine shortage may be involved in platelet activation observed in severe COVID-19. 


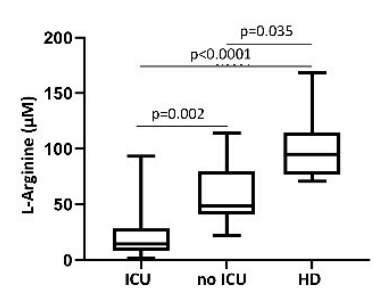

(a)

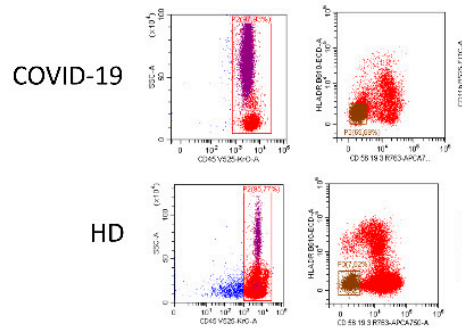

(b)

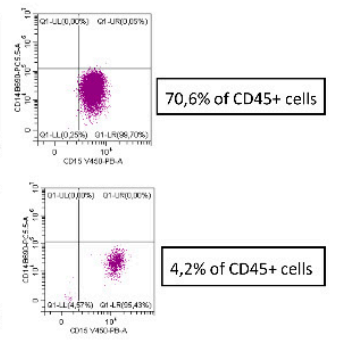

)

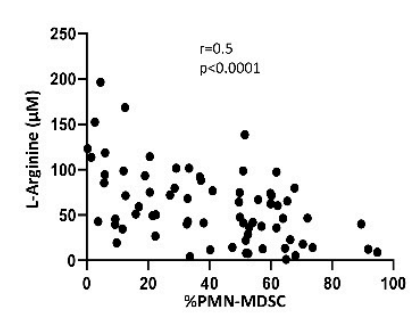

(c)
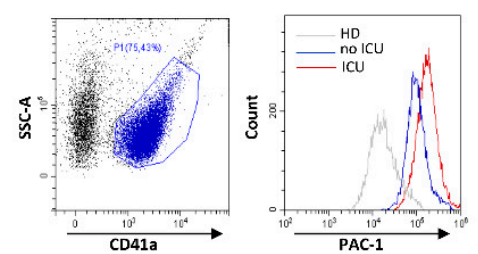

(d)

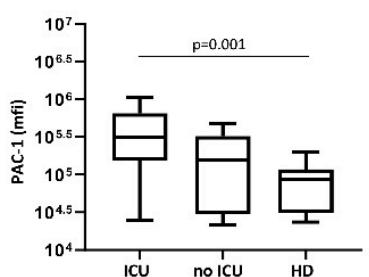

(e)

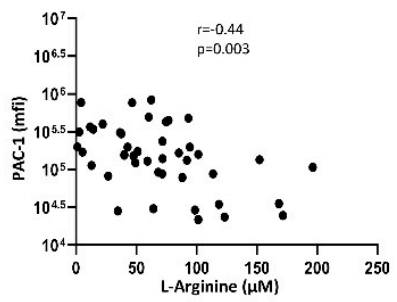

(f)

Figure 1. Plasmatic L-arginine level in SARS-CoV-2 patients correlated with PMN-MDSC frequency. (a) Plasmatic Arginine level of SARSCoV-2 ICU (ICU, $n=31$ ) and no ICU (no ICU, $n=31$ ) patients and healthy donors (HD, $n=9$ ). Results are shown as box and whiskers plot. Kruskal-Walliswith Dunn's post hoc test was applied. (b) Flow-cytometry gating strategy used to identify MDSC among PBMC. Dead cells were excluded by selecting DRAQ7neg cells. One representative COVID-19 patient and one HD are shown. (c) Correlation between plasmatic L-arginine level and PMN-MDSC percentage from SARSCoV-2 infected patients and HD. Non-parametric Spearman correlation was applied. (d) Representative plots of the adopted gating strategy to evaluate platelet activation. Platelets were selected as CD41a+ (SSC/CD41a plot), and PAC-1 expression (mean fluorescence intensity, mfi) was evaluated. (e) PAC-1 expression (mfi) on platelets from ICU $(n=21)$, no ICU $(n=15)$ patients, and HD $(n=9)$. Results are shown as box and whiskers plot. Kruskal-Walliswith Dunn's post hoc test was applied. (f) Correlation between PAC-1 platelet expression (mfi) and L-arginine plasmatic level.

\subsection{PMN-MDSC Induced Platelet Activation by Reducing L-Arginine}

Next, we evaluated whether PMN-MDSCs were directly involved in platelet activation. We found a positive correlation between PAC-1 platelet expression and PMN-MDSC frequency (Figure 2a), suggesting that PMN-MDSC may be involved in arginine deprivation. To confirm that PMN-MDSC may induce platelet activation, we cultured platelet rich plasma (PRP) from HD with PMN-MDSCs isolated from COVID-19 patients and, after $4 \mathrm{~h}$, the expression of PAC-1 on platelets was evaluated by flow cytometry. We found that PMN-MDSCs from COVID-19 were able to activate resting healthy platelets by increasing the expression of PAC-1 (Figure 2b,c). As a control, PBMC depleted from MDSCs were used and no effect was observed. Accordingly, a reduction of L-arginine in the culture supernatants (Figure 2d) was found, indicating that PMN-MDSCs may activate platelets by depriving L-arginine from the microenvironment. 


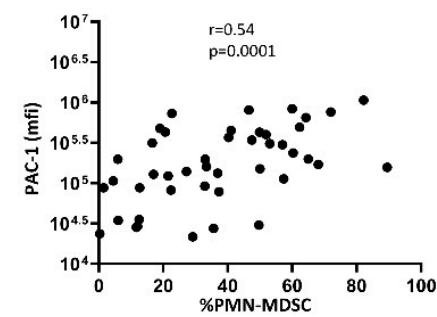

(a)

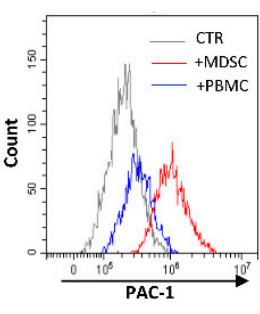

(b)

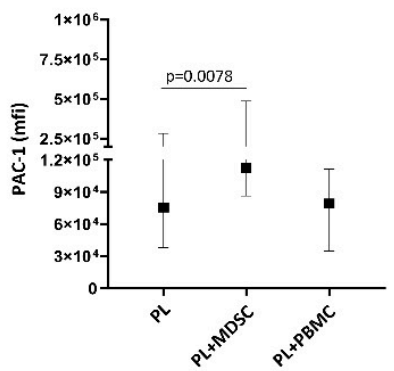

(c)

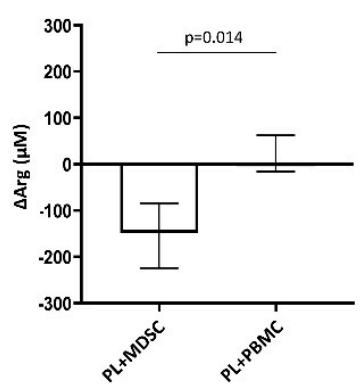

(d)

Figure 2. PMN-MDSC induced platelet activation by reducing L-arginine. (a) Correlation between PAC-1 platelet expression (mfi) and PMN-MDSC percentage from SARSCoV-2 infected patients (ICU $n=21$, no ICU $n=15$ ) and HD ( $n=9$ ). Nonparametric Spearman correlation was applied. (b) Representative histogram plot showing PAC-1 expression on platelets after culture with or without PMN-MDSC or PBMC depleted from MDSCs (PBMC). (c) PAC-1 expression on platelets from HD $(n=6)$ after culture with PMN-MDSC or PBMC from SARSCoV-2 infected patients $(n=6)$. Results are shown as median and IQR. Wilcoxon matched-pairs signed rank test was applied. (d) L-arginine reduction ( $\triangle \mathrm{Arg}$ ) calculated as the difference between L-arginine concentration in the presence of MDSCs or PBMC and platelets alone. Results are shown as median and IQR. A Wilcoxon matched-pairs signed rank test was applied.

\section{Discussion}

Patients with severe COVID-19 commonly present thrombotic disorders, and these conditions have been associated with a higher mortality rate [10]. Moreover, severe COVID-19 is characterized by a strong neutrophilia that persists overtime. Among neutrophils, a strong inflammatory-driven expansion of PMN-MDSCs was observed in severe patients, which significantly reduces the adaptive immune response to SARS-CoV-2 and predicts a fatal clinical outcome $[7,8]$.

In this paper, we analyzed an MDSC function never explored before, to the best of our knowledge, and showed that PMN-MDSCs from COVID-19 patients may be involved in platelet activation by reducing L-arginine availability, highlighting a new interplay between immune regulatory cells and platelet function.

According to previous published data [11], a decrease of L-arginine in the plasma from COVID-19 patients was found. In the present work, we also observed that L-arginine level inversely correlated with PMN-MDSC frequency and with platelet activation. Moreover, PMN-MDSC frequency directly correlated with platelet activation, suggesting a role of PMN-MDSCs in platelet activation during COVID-19. This hypothesis is corroborated by the high level of the enzymes involved in L-arginine catabolism, Arg I, and iNOS, expressed by PMN-MDSCs in COVID-19 patients [7]. Our in vitro experiments further provide a formal proof of the direct role of PMN-MDSC in inducing platelet activation through L-arginine consumption.

Platelet activation has been described during SARS-CoV-2 infection, and it is known to contribute to thromboembolic complications. Besides hyper-inflammation, factors such as a direct SARSCoV-2 infection and antibody-mediated mechanisms have been proposed to contribute to platelet hyperactivity [4]. In the present study, we demonstrated the PMN-MDSC as a new player in platelet homeostasis, highlighting an unprecedented function of the PMN-MDSC during COVID-19. Correlations between PMN-MDSC frequency with L-arginine concentration and with platelet activation have been shown during other infections such as severe fever caused by a bunyavirus [12]. Herein, we demonstrated that PMN-MDSC, by decreasing L-arginine, might directly contribute to platelet activation, shedding light on a novel role of PMN-MDSCs besides immune suppression.

\section{Conclusions}

Our findings demonstrate the direct involvement of PMN-MDSCs in platelet activation during COVID-19, confirming the MDSC expansion as one of the main events driving 
COVID-19 pathogenesis. These results also reveal new therapeutic perspectives targeting MDSC number and function, a promising strategy already under evaluation in cancer patients $[13,14]$.

Author Contributions: A.S. and C.A. contributed to conceptualization; A.S. and C.A. contributed to experimental design; G.G. (Germana Grassi), S.G., V.B., E.C. and R.C. contributed to flow cytometry experiments and analysis; A.S., G.G. (Germana Grassi), S.N., E.T. and D.M. contributed to functional experiments; A.S. performed statistical analysis; L.M., E.N., G.G. (Gabriele Garotto) and A.B. contributed to patient management; A.S., G.G. (Germana Grassi), and C.A. wrote the paper; M.B. and G.I. contributed to revising the paper. All authors have read and agreed to the published version of the manuscript.

Funding: This research was funded by the Italian Ministry of Health [Ricerca Corrente-Linea 1, COVID-2020-12371735 and COVID-2020-12371817].

Institutional Review Board Statement: The study was conducted according to the guidelines of the Declaration of Helsinki and approved by the Institutional Review Board of National Institute for Infectious Diseases “Lazzaro Spallanzani” (approval number: 9/2020).

Informed Consent Statement: Informed consent was obtained from all subjects involved in the study.

Data Availability Statement: Publicly available datasets were analyzed in this study. This data can be found here: [https://rawdata.inmi.it/].

Conflicts of Interest: The authors declare no conflict of interest. The funders had no role in the design of the study; in the collection, analyses, or interpretation of data; in the writing of the manuscript; or in the decision to publish the results.

\section{References}

1. Borges do Nascimento, I.J.; Cacic, N.; Abdulazeem, H.M.; von Groote, T.C.; Jayarajah, U.; Weerasekara, I.; Esfahani, M.A.; Civile, V.T.; Marusic, A.; Jeroncic, A.; et al. Novel coronavirus infection (COVID-19) in humans: A scoping review and meta-analysis. J. Clin. Med. 2020, 9, 941. [CrossRef] [PubMed]

2. Bilaloglu, S.; Aphinyanaphongs, Y.; Jones, S.; Iturrate, E.; Hochman, J.; Berger, J.S. Thrombosis in hospitalized patients with COVID-19 in a New York City health system. JAMA 2020, 324, 799-801. [CrossRef] [PubMed]

3. Tang, N.; Li, D.; Wang, X.; Sun, Z. Abnormal coagulation parameters are associated with poor prognosis in patients with novel coronavirus pneumonia. J. Thromb. Haemost. 2020, 18, 844-847. [CrossRef] [PubMed]

4. Shen, S.; Zhang, J.; Fang, Y.; Lu, S.; Wu, J.; Zheng, X.; Deng, F. SARS-CoV-2 interacts with platelets and megakaryocytes via ACE2-independent mechanism. J. Hematol. Oncol. 2021, 14, 72. [CrossRef] [PubMed]

5. Pinto, V.L.; de Souza, P.F.; Brunini, T.M.; Oliveira, M.B.; Moss, M.B.; de Sá Siqueira, M.A.; Ferraz, M.R.; Mendes-Ribeiro, A.C. Low plasma levels of L-arginine, impaired intraplatelet nitric oxide and platelet hyperaggregability: Implications for cardiovascular disease in depressive patients. J. Affect. Disord. 2012, 140, 187-192. [CrossRef] [PubMed]

6. Greten, T.F.; Manns, M.P.; Korangy, F. Myeloid derived suppressor cells in human diseases. Int. Immunopharmacol. 2011, 11, 802-807. [CrossRef]

7. Sacchi, A.; Grassi, G.; Bordoni, V.; Lorenzini, P.; Cimini, E.; Casetti, R.; Tartaglia, E.; Marchioni, L.; Petrosillo, N.; Palmieri, F.; et al. Early expansion of myeloid-derived suppressor cells inhibits SARS-CoV-2 specific T-cell response and may predict fatal COVID-19 outcome. Cell Death Dis. 2020, 11, 921. [CrossRef] [PubMed]

8. Falck-Jones, S.; Vangeti, S.; Yu, M.; Falck-Jones, R.; Cagigi, A.; Badolati, I.; Österberg, B.; Lautenbach, M.J.; Åhlberg, E.; Lin, A.; et al. Functional monocytic myeloid-derived suppressor cells increase in blood but not airways and predict COVID-19 severity. $J$. Clin. Investig. 2021, 131. [CrossRef]

9. Lee, B.R.; Chang, S.Y.; Hong, E.H.; Kwon, B.-E.; Kim, H.M.; Kim, Y.-J.; Lee, J.; Cho, H.-J.; Cheon, J.-H.; Ko, H.-J. Elevated endoplasmic reticulum stress reinforced immunosuppression in the tumor microenvironment via myeloid-derived suppressor cells. Oncotarget 2014, 5, 12331-12345. [CrossRef]

10. Levi, M.; Thachil, J.; Iba, T.; Levy, J.H. Coagulation abnormalities and thrombosis in patients with COVID-19. Lancet Haematol. 2020, 7, e438-e440. [CrossRef]

11. Reizine, F.; Lesouhaitier, M.; Gregoire, M.; Pinceaux, K.; Gacouin, A.; Maamar, A.; Painvin, B.; Camus, C.; Le Tulzo, Y.; Tattevin, P.; et al. SARS-CoV-2-induced ARDS associates with MDSC expansion, lymphocyte dysfunction, and arginine shortage. J. Clin. Immunol. 2021, 41, 515-525. [CrossRef] [PubMed]

12. Li, X.K.; Lu, Q.B.; Chen, W.W.; Xu, W.; Liu, R.; Zhang, S.-F.; Du, J.; Li, H.; Yao, K.; Zhai, D.; et al. Arginine deficiency is involved in thrombocytopenia and immunosuppression in severe fever with thrombocytopenia syndrome. Sci. Transl. Med. $2018,10$. [CrossRef] [PubMed] 
13. Eriksson, E.; Wenthe, J.; Irenaeus, S.; Loskog, A.; Ullenhag, G. Gemcitabine reduces MDSCs, tregs and TGFbeta-1 while restoring the teff/treg ratio in patients with pancreatic cancer. J. Transl. Med. 2016, 14, 282. [CrossRef] [PubMed]

14. Tobin, R.P.; Jordan, K.R.; Robinson, W.A.; Davis, D.; Borges, V.F.; Gonzalez, R.; Lewis, K.D.; McCarter, M.D. Targeting myeloidderived suppressor cells using all-trans retinoic acid in melanoma patients treated with Ipilimumab. Int. Immunopharmacol. 2018, 63, 282-291. [CrossRef] [PubMed] 